\title{
"Mutig werden kann ich lernen": Kognitive Verhaltenstherapie bei ausgeprägter Angstsymptomatik, Entwicklungsstörung und Intelligenzminderung
}

\author{
Susanne Knappe ${ }^{a, b}$ \\ anstitut für Klinische Psychologie und Psychotherapie, Technische Universität Dresden, Dresden, Deutschland; \\ bInstitutsambulanz und Tagesklinik für Psychotherapie, IAP-TU Dresden GmbH, Dresden, Deutschland
}

\section{Schlüsselwörter}

Psychotherapie · Kognitive Verhaltenstherapie · Geistige Behinderung $\cdot$ Angst

\section{Zusammenfassung}

Hintergrund: Die psychotherapeutische Behandlung von Kindern und Jugendlichen mit geistiger Behinderung unterliegt einer Vielzahl von Barrieren. Fallbericht: Der hier vorgestellte Behandlungsfall illustriert die erfolgreiche Anwendung kognitiver Verhaltenstherapie bei einem 10-jährigen Mädchen bei Entwicklungsstörung und Intelligenzminderung. Nach einer medizinisch notwendigen Untersuchung entstanden ausgeprägte Angstsymptome und Verhaltensprobleme. Infolge einer bekannten Epilepsie war der Einsatz expositionsbasierter Techniken fraglich. Die kognitive Verhaltenstherapie umfasste 64 Behandlungseinheiten. Das Vorgehen zur Selbstbeobachtung, Rationalvermittlung und Angstbewältigung war stark handlungsbezogen und wenig kognitiv orientiert und fand im häuslichen Setting mit Einbezug der Familienmitglieder statt. Nachfolgend wurden auch aufgrund der zunehmenden Reifung des Kindes - kognitiv anspruchsvollere Techniken eingesetzt. Zur 38. Behandlungseinheit wurden zuvor angstbesetzte Situationen dem Entwicklungsstand angemessen und selbstständig bewältigt. Eine nachfolgende medizinische Untersuchung ähnlich der Auslösesituation wurde therapeutisch vorbereitet und sicher bewältigt. Zu Behandlungsende waren die Alltagskompetenzen maßgeblich erweitert. Schlussfolgerungen: Entgegen der Vorannahmen bedurfte es nur weniger Anpassungen an das Setting und die Behandlungstechniken im
Vergleich zum Vorgehen bei ähnlicher Symptomatik ohne geistige Behinderung. Durch die psychotherapeutische Behandlung konnte kurz- und langfristig eine Manifestation (Eskalation) von weiterem Problemverhalten verhindert werden. Das Erkennen und die Auseinandersetzung mit dem "Anderssein" infolge der geistigen Behinderung und verfügbarer Alltagskompetenzen bergen Entwicklungsrisiken und -chancen, die durch eine psychotherapeutische Begleitung gut bewältigt werden können. @ 2021 The Author(s)

Published by S. Karger AG, Basel

\section{"Learning to Be Brave": Cognitive-Behavioral Therapy in a Case with Severe Anxiety, Developmental Disorder and Intellectual Disabilities}

\section{Keywords}

Psychotherapy · Cognitive-behavioral therapy · Mental retardation - Anxiety

\section{Abstract}

Objectives: Psychotherapy in children and adolescents with intellectual disabilities is hampered by a variety of barriers. Case Description: This case illustrates the successful cognitive-behavioral therapy (CBT) in a 10-yearold girl with anxiety, developmental disorder and intellectual disabilities. Severe anxiety symptoms and behavioral problems manifested after a medically inevitable examination. Because of a known epilepsy, administration of exposure-based techniques was questionable. The mercial purposes requires written permission. 
CBT comprised 64 therapy sessions. Interventions on self monitoring, introducing the treatment rationale and anxiety coping were oriented towards observable behaviors rather than towards self-reflection. Sessions were first conducted at the patient's home, including involvement of family members. With increasing maturation of the patient, cognitive techniques were applied as well. After 38 sessions, the patient was able to manage anxiety-provoking situations without avoidance behaviors, according to her developmental age. A medical examination, similar to the situation of anxiety onset, was trained in advance and was then successfully managed. Finally, competences of daily living have substantially increased. Conclusions: Only minor adaptations to the treatment setting and techniques were needed, as compared to treatments in cases with similar anxiety but without intellectual disabilities. Here, psychotherapy most likely prevented the escalation of problem behaviors in the long term. Dealing with "being different" due to intellectual disabilities and limited competences in daily living implies developmental risks and chances, which both can be successfully mastered with psychotherapeutic support.

C 2021 The Author(s)

Published by S. Karger AG, Basel

\section{Einleitung}

Kinder und Jugendliche mit geistiger Behinderung leiden 3- bis 5-mal häufiger an psychischen Störungen im Vergleich zur Durchschnittsbevölkerung [Deb et al., 2001; Sarimski, 2007]; die Zahl der Verhaltensprobleme ist etwa um das 10-fache höher [Schützwohl et al., 2016]. Das erhöhte Erkrankungsrisiko für psychische Störungen im Vergleich zu Gleichaltrigen ohne geistige Behinderung wird unter anderem auf biologisch-genetische Einflussfaktoren sowie außerordentliche psychosoziale Belastungen zurückgeführt. Möglicherweise treten belastende Ereignisse besonders häufig bei Menschen mit geistiger Behinderung auf, weil sie eine erhöhte Verletzlichkeit haben, ihnen protektive Faktoren fehlen oder sie Probleme in der Bewältigung widriger Umstände haben [Jansen et al., 2002].

Hier erscheinen psychotherapeutische Maßnahmen als hilfreiches Versorgungsangebot zur Minderung von Verhaltensproblemen und Verbesserung der Alltagskompetenzen [Bergmann, 2019]. Allerdings wird eine Unterversorgung für Kinder mit geistiger Behinderung im Bereich der Kinder- und Jugendlichenpsychotherapie verzeichnet [Werther 2005; Hennicke, 2013; DGKJP, 2014], bemessen anhand des epidemiologisch geschätzten Behandlungsbedarfs und der tatsächlich vorhandenen Behandlungskapazitäten [Metaxas et al., 2014]. Neben strukturellen Barrieren für die Bereitstellung und Inanspruchnahme (spezialisierter) psycho- therapeutischer Maßnahmen mögen fachliche, organisatorische und zeitliche Anforderungen an die Diagnostik und Psychotherapie, Schwierigkeiten bei der Therapiebewilligung wie z.B. geistige Behinderung als Ablehnungsgrund oder Verweis auf Behandlung als genuiner Auftrag für (sozial)pädagogische Institutionen sowie eine fehlende Verankerung des Themas in bestehenden Ausbildungscurricula eine Rolle spielen [Jäckel und Simon, 2014; Metaxas et al., 2014; Stünkel-Grees et al., 2018]. Ein vermindertes Sprachverständnis, eingeschränkte oder unkonventionelle Ausdrucksmöglichkeiten aufseiten der Patienten können die Kommunikation erschweren.

Für die psychotherapeutische Behandlung von Kindern und Jugendlichen mit geistigen Behinderungen gibt es keine Begrenzung auf ein bestimmtes Richtlinienverfahren oder Techniken; im Versorgungsalltag überwiegen spieltherapeutische Techniken, Musik- und kunsttherapeutische Ansätze [Stünkel-Grees et al., 2018]. Überzeugende Wirksamkeitsnachweise für die psychotherapeutische Behandlung von Kindern und Jugendlichen mit geistiger Behinderung fehlen [Vereenooghe und Langdon, 2013; Ali et al., 2015]. In Einzelfall- und nichtkontrollierten Studien [Didden et al., 2006; Sappok et al., 2010; Hennicke, 2011] wurden u.a. für Verhaltenstrainings bei phobischer Vermeidung [Jennett und Hagopian, 2008] und für die psychotherapeutische Behandlung von posttraumatischer Belastungsstörung [Mevissen und de Jongh, 2010; Zeiger et al., 2017] positive Effekte gefunden. Darüber hinaus werden Effekte für Therapieansätze der dritten Welle [Robertson, 2011; Elstner, 2016] berichtet. Umfassende empirische Effektivitätsnachweise wurden in erster Linie für den Einsatz verhaltenstherapeutischer Verfahren bei Erwachsenen mit geistiger Behinderung publiziert [Didden et al., 1997; Willner, 2005; Prout und Browning, 2011; Sturmey, 2012; Vereenooghe und Langdon, 2013; DGKJP, 2014]. Dennoch steht einer zunehmenden Zahl von Studien über die Effizienz von Psychotherapie für Menschen mit geistiger Behinderung eine sehr geringe Zahl von Therapeuten gegenüber, die über eine hinreichende Bereitschaft und Qualifikation zur Behandlung von Menschen mit geistiger Behinderung verfügen [Metaxas et al., 2014].

In dem hier vorgestellten Fall werden Behandlungselemente und das Behandlungsergebnis einer kognitiven Verhaltenstherapie bei einem 10-jährigen Mädchen mit einer Angststörung und einer kombinierten umschriebenen Entwicklungsstörung beschrieben. Zudem bestanden eine mittelgradige Intelligenzminderung und eine bekannte Epilepsie. Damit soll die Machbarkeit für die psychotherapeutische Behandlung von Kindern und Jugendlichen mit geistiger Behinderung illustriert werden. 


\begin{tabular}{|c|c|c|}
\hline & S & $\begin{array}{l}\text { Am Nachmittag nach der Schule. Pat. vespert in Anwesenheit der Mutter in der Küche. Vater ist noch dienstlich unterwegs, der Bruder beim } \\
\text { Fußballtraining. Mutter kündigt an „Ich bringe kurz den Müll nach draußen“. }\end{array}$ \\
\hline & $\mathrm{O}$ & Entwicklungsverlauf abhängig von der BNS-Epilepsie. Entwicklungsverzögerung. Intelligenzminderung. Hemianopsie rechts \\
\hline \multirow{4}{*}{$\begin{array}{l}\mathrm{R} \\
\mathrm{e} \\
\mathrm{a} \\
\mathrm{k} \\
\mathrm{ti} \\
\mathrm{o} \\
\mathrm{n}\end{array}$} & $\mathbf{R}_{\mathrm{kog}}$ & „Was? Nein, das geht nicht. Da bekomme ich Angst." \\
\hline & Rphys & Unruhig, angespannt, zunehmende Erregung \\
\hline & Remot & ängstlich-besorgt, starkes Unwohlsein \\
\hline & $R_{\text {mot }}$ & "Mama geh nicht", „nicht rausgehen“, die Mutter festhalten \\
\hline \multirow{7}{*}{$\begin{array}{l}\mathrm{C} \\
\mathrm{o} \\
\mathrm{n} \\
\mathrm{s} \\
\mathrm{e} \\
\mathrm{q} \\
\mathrm{u} \\
\mathrm{e} \\
\mathrm{n} \\
\mathrm{z}\end{array}$} & $\mathrm{C}+$ kurz & mütterliche Zuwendung \\
\hline & $\epsilon_{\text {+kurz }}$ & Alltagsaufgaben können nicht erledigt werden (Mutter) \\
\hline & $\epsilon_{\text {kur }}$ & Angst und Anspannung werden vermieden (Mutter und Kind), kurzzeitig Symptomlinderung (Kind) \\
\hline & $\epsilon_{\text {lang }}$ & Besuche bei anderen Kindern, Nachbarn oder Spielen im Wohngebiet gelingen nicht mehr (Verlust sozialer Verstärker) \\
\hline & C-lang & Selbstwirksamkeitserleben fehlt, Trennungssituationen von den Eltern werden nicht entwicklungs-/altersangemessen bewältigt, \\
\hline & & $\begin{array}{l}\text { bewältigt werden. Belastung der familiären Beziehungen (Einschränkungen der Eltern, Verantwortungsübernahme wird zunehmend auch } \\
\text { vom Bruder verlangt) }\end{array}$ \\
\hline & K & $\begin{array}{l}\text { Kontiguität zwischen Situation und Reaktion hoch, Kontiguität zwischen Reaktion und Konsequenzen hoch } \\
\text { Kontingenz zwischen Situation und Reaktion hoch, Kontingenz zwischen Reaktion und Konsequenzen hoch }\end{array}$ \\
\hline
\end{tabular}

Abb. 1. SORCK-Modell zur Angstsymptomatik von Lissy, das Grundlage für die Fallkonzeption und Behandlungsplanung war.

\section{Fallbeschreibung}

Alle erwähnten Personen gaben ihre Zustimmung zu ihrer Einbeziehung in die Publikation. Die Namen und identifizierende Personenmerkmale wurden geändert.

\section{Zuweisungsanlass}

Die 10-jährige Lissy wird auf Initiative der leiblichen Mutter in der Hochschulambulanz vorgestellt. Seit der operativen Entfernung eines Ganglioglioms im Herbst 2015 und einer ungünstig verlaufenen Nachuntersuchung 2016 habe Lissy ausgeprägte Angst, wenn die Mutter oder ein anderes Familienmitglied das Haus kurz verlasse, um in den Garten zu gehen oder den Müll fortzubringen. Lissy fange dann an zu weinen, laut zu rufen bis hin zum Schreien, stampfe mit den Füßen auf und gerate außer sich. Keinem Familienmitglied sei es daher noch möglich, z.B. auf der Terrasse zu sitzen oder sich im Garten aufzuhalten. Lissy fordere beharrlich, dass alle Familienmitglieder im Haus und in Sichtweite seien. Ähnlich sei es Lissy nicht mehr möglich, zum Spielen zu den Nachbarskindern (in Sichtweite zum Elternhaus) oder zu einem nahegelegenen Bauernhof zu gehen. Die beschriebene Problematik sei auf das häusliche Umfeld beschränkt; Lissy zeige keine Angstsymptome während des Schulbesuches oder in anderen Lebensbereichen. Anliegen der Familie ist eine Besserung der beschriebenen Beschwerden; Lissy möge zum präoperativen $\mathrm{Zu}$ stand der beschriebenen Alltagskompetenzen zurückfinden.

\section{Eigenanamnese}

Lissy wächst als jüngeres Kind (Bruder, +3 Jahre) bei den leiblichen Eltern (Mutter 40 Jahre, Angestellte; Vater 53 Jahre, Angestellter) auf. Das Elternhaus ist geprägt durch einen liebevollen Umgang miteinander und elterliche Fürsorge; kindliche Bedürfnisse nach Versorgung, Zuwendung und Sicherheit werden altersund entwicklungsangemessen bedient. Die Familie erscheint in ihren Beziehungen zueinander eng und wohlwollend. Die Mutter als wichtigste Bezugsperson beschreibt Lissy als liebevoll, mitfühlend und fröhlich. Die Schwangerschaft, Entbindung und frühkindliche Entwicklung verliefen unauffällig bis etwa zum 6. Le- bensmonat. Mit Beginn einer Blitz-Nick-Salaam-Epilepsie (WestSyndrom; kurz: BNS-Epilepsie) im ersten Lebensjahr wurden motorische Entwicklungsrückschritte beobachtet. Die weitere Entwicklung sei kontinuierlich, wenn auch verzögert verlaufen. Lissy besucht seit dem 7. Lebensjahr eine Schule mit dem Förderschwerpunkt geistige Entwicklung. In ihrer Freizeit spielt Lissy gerne mit Playmobil, Puppen, fährt Fahrrad, malt, schwimmt, singt. Das Entwicklungsalter zur Erstvorstellung entspricht dem eines reifen Vorschulkindes.

\section{Spezielle Anamnese}

Lissy leidet seit dem 6. Lebensmonat unter einer symptomatisch fokalen Epilepsie links temporal, bedingt durch ein Gangliogliom sowie eine fokale kortikale Dysplasie. Präoperativ traten bis zum 9. Lebensjahr 3-4 Krampfanfälle täglich mit Beteiligung der Amygdala, Hippocampus und Ausdehnung in die untere Insel, den Temporallappenstiel, den hinteren Thalamus und kleine Anteile des Mittelhirns auf. Das Gangliogliom wurde im 9. Lebensjahr epilepsiechirurgisch in Form einer Resektion des Temporal- und Occipitallappens links unter Aussparung des möglichen Wernicke-Areals und unter Belassung der tumorösen Anteile im hinteren Thalamus sowie im Mittelhirn entfernt. Ein Befall oder maligne Veränderung amygdalaer Strukturen wurden nicht beobachtet.

\section{Präoperativer Zustand und auslösende Situation}

Infolge der BNS-Epilepsie und der einhergehenden Anfallssituation waren die allgemeine Belastbarkeit, Aufmerksamkeit und Ausdauer von Lissy vermindert, Ablenkbarkeit und Impulsivität erhöht. Präoperativ reagierte Lissy auf Irritation oder Frustration im Alltag mit exzessivem Weinen, Jammern oder Anklammern. Die Familie versuchte dies weitestgehend zu vermeiden, um eine Intensivierung des Erregungszustandes und damit eventuell ein erneutes Anfallsgeschehen zu reduzieren. Vorranging aufseiten der Mutter (und in weniger starkem Ausmaß aufseiten des Vaters) entstanden Vermeidungsverhaltensweisen, z.B. Trennungssituationen vermeiden, Unruhe und Aufregung vermeiden. Zur kurzfristigen Linderung von Erregungszuständen im Alltag trugen zudem regelmäßige Erholungspausen und ein täglicher Mittagsschlaf bei. 
Es wurde einer Besserung dieser Beeinträchtigungen nach der Operation im Herbst 2015 erwartet. Dieser Operation folgte eine Reihe von Nachuntersuchungen im Januar, Februar, Juni und September 2016 mittels MRT und unter Sedierung, denen Lissy zunehmend ängstlich gegenüberstand.

Nach der letzten Nachuntersuchung im September 2016 erwachte Lissy in einer fremden Umgebung und umgeben von (fremdem) Pflegepersonal. Sie reagierte mit Irritation und Unruhe und rief nach ihrer Mutter, die jedoch aufgrund pflegerischer Maßnahmen noch nicht zu Lissy gelangte. Lissy erlebte eine weitere Steigerung der Unruhe und Erregung (ohne Anfallsgeschehen), die erst nach Wiedervereinigung mit der Mutter spürbar absank. Für Lissy und ihre Mutter war diese Erfahrung derart prägend, dass die Mutter ihr Vermeidungsverhalten fortsetzte, um jegliche Unruhe und Aufregung zu vermeiden (negative Verstärkung), und dass Lissy den Sichtkontakt zur Mutter bzw. anderen Familienmitgliedern einforderte. Es gelang Lissy und ihrer Familie nicht, die bisherigen Verhaltensweisen (Rückversicherung, Verstärkungsverhalten) aufzugeben.

Diagnostische Maßnahmen und diagnostische Einordnung

Die psychologische Diagnostik umfasste einen psychopathologischen Befund zur Erstvorstellung, Verhaltensproben und Fremdangaben im häuslichen Kontext, Selbstauskünfte von Lissy sowie ein kinderärztliches und neurologisches Konsil.

Psychopathologischer Befund zur Erstvorstellung. Lissy war in der Interaktion offen, sprach laut, teilweise leicht verwaschen und undeutlich. Wortschatz und Wortflüssigkeit erschienen durchschnittlich. Sie war vollständig orientiert hinsichtlich Zeit, Ort, Situation und Person. Motorisches Verhalten war weitestgehend unauffällig. Keine Auffälligkeiten in Bezug auf formales und inhaltliches Denken, Aufmerksamkeit, und Gedächtnis. Intelligenz grob orientiert unterhalb des Normbereiches. Stimmung/Affekt unbeeinträchtigt und schwingungsfähig. Suizidalität nicht erkennbar. Keine Hinweise auf selbstverletzendes Verhalten oder Fremdgefährdung.

Verhaltensproben im häuslichen Kontext. Lissy bewohnt ein eigenes Kinderzimmer im obersten Stock des Hauses, kann von dort teilweise in den Garten sehen. Im Erdgeschoss befindet sich eine Terrasse, die die Familie auf Wunsch von Lissy nicht betreten soll ("Ich will euch sehen!"). Der Zugang zum Garten erfolgt über ein Gartengeschoss. Ein Müllplatz befindet sich seitlich zur fensterlosen Fassade und ist nicht einsehbar vom Haus. Innerhalb des Hauses können sich alle Familienmitglieder ohne Angsterleben von Lissy frei bewegen, sofern Sichtkontakt besteht. Verlässt ein Familienmitglied das Haus (angekündigt) für kurze Wege (zum Nachbarn, zum Müllplatz, in den Garten) oder gerät außer Sichtweite, reagiert Lissy mit Weinen, Rufen, Schreien, gesteigerter Erregung (Abb.1).

Selbstauskünfte der Patientin. Eine Beurteilung anhand des Bochumer Angstinventars [BAV 3-11; Mackowiak und Lengning, 2010] erbrachte keine Hinweise auf spezifische oder soziale Ängste. Primäre Angstregulationsstrategien sind Problemvermeidung und Einholen sozialer Unterstützung durch Familienmitglieder.

Kinderärztliches Konsil. Unauffällig. Keine Kontraindikation für psychotherapeutische Behandlung. Hinweis auf neurologische Mitbehandlung. Medikamentöse Begleitbehandlung mit Timoxsaft $^{\circledR} 10 \mathrm{~mL}(1-0-1)$ und Lamotrigin ${ }^{\circledR} 50(2-0-2)$.

Neurologisches Konsil. Seit dem operativen Eingriff wurden keine Krampfanfälle mehr beobachtet; allerdings besteht seitdem eine Hemianopsie rechts. Aufgrund des Gesichtsfeldausfalles geraten Bezugspersonen schneller aus dem Blickwinkel von Lissy, worauf sie mit Unruhe und Unsicherheit/Angst reagiert.

Postoperative Intelligenz- und entwicklungsbezogene Befunde. Im HAWIK-III [Tewes et al., 1999] wurden keine bedeutsamen Veränderungen von prä- zu postoperativ beobachtet (Handlungs-
$\mathrm{IQ}=46$, verbaler IQ $=63$ ). Verbale Kurzzeitmerkspanne unterdurchschnittlich, verbales Arbeitsgedächtnis leicht unterdurchschnittlich, visuelle Kurzzeitmerkspanne weit unterdurchschnittlich. Postoperativ wurden deutliche Veränderungen in den Verhaltensauffälligkeiten [Vineland Maladaptive Behavior Scale, Sparrow et al., 1984] berichtet; es gab nur noch leichte Abweichungen gegenüber entwicklungs- bzw. altersgleicher Kinder. Auch die Erholungspausen waren postoperativ nicht mehr notwendig, da die Belastbarkeit und Ausdauer deutlich gebessert waren. Aufmerksamkeit und Konzentration waren gesteigert, Fortschritte ergaben sich in der Alltagsselbstständigkeit und in den Sozialkompetenzen entsprechend einem Entwicklungsniveau $4^{1 / 2}$ bis $4^{3 / 4}$ Jahre alter Kinder.

\section{Diagnose und differentialdiagnostische Einordung}

Die berichtete Angstsymptomatik wurde nach ICD-10 als sonstige phobische Störung (F 40.8) kodiert. Auf den weiteren Achsen [Remschmidt et al., 2006] wurden kodiert: F83 kombinierte umschriebene Entwicklungsstörung (hier: Entwicklungsverzögerung von Sprache und Motorik) (Achse II), F71 mittelgradige Intelligenzminderung (Achse III), G40.X fokale Epilepsie links temporal (Achse IV), keine abweichenden psychosozialen Umstände (Achse V) und ernsthafte Beeinträchtigung in den meisten Bereichen, benötigt Aufsicht oder Betreuung (Achse VI). Die krankheitswertige Symptomatik entsprach am ehesten einer Trennungsangststörung (F93.0), wobei das ICD-10-Kriterium C nicht erfüllt war. Auch eine phobische Störung im Kindesalter (F93.1) konnte aufgrund der bekannten Entwicklungsverzögerung nicht kodiert werden. Ungeachtet der diagnostischen Einordung war mit hoher Wahrscheinlichkeit anzunehmen, dass ohne Behandlung eine ungünstige Symptomprogression eintritt. Durch eine Behandlung sollten zudem die Entwicklung sekundärer Komplikationen (Fortschreiten der Angstsymptomatik, Entwicklung einer komorbiden depressiven Symptomatik; Einschränkungen und Belastung der Familienmitglieder) unterbunden und die Alltagskompetenzen weiter verbessert werden.

\section{Behandlungsziele und Behandlungsplan}

Es erschien nach Rücksprache mit den Kolleginnen der psychotherapeutischen Ambulanz sowie den Kollegen der Neurologie umsetzbar, im Rahmen eines Trainings zur Angstbewältigung alters- und entwicklungsgerechte Alltagsfertigkeiten und Selbstständigkeit ("im Haus bleiben, wenn Mama den Müll fortbringt”) wiederzuerlangen. Lissy formulierte als oberstes Therapieziel "Mut haben im Haus". Sie baute rasch guten und belastbaren Kontakt zur Behandlerin auf. Prognostisch günstig war, dass Lissy beschult wurde, mit Token umgehen konnte und auf diese Weise bereits "ohne Angst allein 
schlafen im Zimmer" erlernt hatte. Zu Therapiebeginn war Lissy mehr als 12 Monate anfallsfrei. Die Behandlung war gutachterpflichtig. Beantragt und genehmigt wurden zunächst $25+6$ Behandlungseinheiten, im Verlauf erfolgte eine Umwandlung in eine Langzeitbehandlung (35 $+8)$. Letztlich fanden 64 Behandlungseinheiten mit Einbezug der Bezugspersonen statt.

\section{Behandlungsverlauf und Ergebnisse April 2017 bis Juni 2018 (1.-38. Behandlungseinheit)}

Zunächst wurden mit Hilfe von Selbst- und Fremdbeobachtungen die verhaltens- und bedingungsanalytischen Zusammenhänge des Problemverhaltens bei Trennungen von den Eltern bzw. Alleinsein gemeinsam mit Lissy direkt im häuslichen Umfeld kindgerecht erfasst. Eine angebotene Psychoedukation zu den Themen "Was ist Angst?", "Wozu ist Angst gut?", "Wann macht Angst krank?" irritierte Lissy; stattdessen war sie am "Mutig werden" interessiert. Daher wurde die Psychoedukation nicht weiter verfolgt.

Um jene angstbesetzten Situationen zu bestimmen, in denen Lissy mutig werden möchte, wurden gemeinsam mit Lissy Situationen und ihre Variationen (mit/ohne Sichtkontakt, Entfernungen, mit/ohne therapeutische Begleitung) in aufsteigender Reihenfolge aufgelistet.

\section{Beispiele für angstbesetzte Situationen von Lissy}

- In der Küche sitzen, wenn Mama den Müll fort bringt

- Auf einer anderen Etage im Haus sein als andere Familienmitglieder

- Den Balkon allein betreten

- Allein im Garten sein/zu den Hasen gehen

- Löwenzahn holen (Wiese gegenüber dem Elternhaus)

- Roller fahren im Wohnviertel

- Allein im Auto warten, während die Eltern eine kurze Besorgung erledigen

- Den Spielplatz nutzen (gegenüber dem Elternhaus)

- Die Hühner des Nachbarn besuchen

Primäres Behandlungsziel war das Aufsuchen der angstbesetzen Situation und die erfolgreiche Bewältigung. Das Behandlungsrational wurde anhand einer Helferfigur vermittelt ["Mutig werden kannst du lernen", die Geschichte von Til Tiger aus Ahrens-Eipper und Leplow, 2004], die Expositionen erfolgten in vivo und graduiert. Lissy dokumentierte jede Exposition selbstständig, z.B. durch schrittweises Ausmalen eines Til-Tiger-Bildes (Selbstverstärkung), zusätzlich wurde ein Tokensystem bei den ersten Expositionen eingesetzt (extrinsische Belohnung durch Sticker, Videos und Fotos). Alle Behandlungstermine fanden im häuslichen Setting und mit Beteiligung der Familienmitglieder in den Expositionen (z.B. wiederholtes Verlassen der Küche, der Etage, des
Hauses) statt. Die Expositionen folgten einem regelhaften Ablauf:

1. Festlegung des heutigen Trainingsziels ( $5 \mathrm{~min}$ allein in der Küche bleiben) anhand der Angsthierarchie

2. Einstimmung auf das Training durch Wiederholung der letzten Stunde (z.B. 3 min allein in der Küche bleiben)

3. Vorbereitung von alternativen Verhaltensweisen, zunächst angeleitet, dann zunehmend selbstständig (Bereitstellung von Malbedarf, Hörbuch, Spielmaterialien o.ä.)

4. Verabschiedung der Therapeutin (individueller Handgruß)

5. Rückkehr der Therapeutin, Lob und Bestärkung Im Rahmen der Elternarbeit wurden psychoedukative Inhalte zur Reduktion von Sicherheits- und Vermeidungsverhalten eingesetzt und die Durchführung des Angstbewältigungstrainings ("Wir trainieren mutig zu werden") in Form von Hausaufgaben an Lissys Eltern übergeben. In keiner Exposition trat eine gesteigerte Erregung auf: die Situationen wurden anfangs derart kleinschrittig bearbeitet, dass eine erhöhte Anspannung, aber keine übersteigerte Angstreaktion einsetzte. Der Einsatz von Token wurde kontinuierlich reduziert. Zur 35. Behandlungseinheit konnte Lissy zuvor angstbesetzte Situationen innerhalb und außerhalb des Elternhauses (Roller fahren im Wohnviertel, auf Eltern im Auto warten) wieder ihrem Entwicklungsstand angemessen und selbstständig bewältigen. Lissy konnte ihre Alltagskompetenzen somit deutlich erweitern und sie wurde selbstbewußter und stolz auf sich (z.B. "Ich schaffe es, auf Mama zu warten").

\section{Erste Ergänzung zum Behandlungsplan: Bewältigung einer MRT-Untersuchung von August 2017 bis September 2017 (39.-45. Behandlungseinheit)}

Die geplante Nachkontrolle des Operationsergebnisses mittels Schädel-MRT (geplant Oktober 2017) führte zu einem Wiederauftreten erheblicher Ängste und Vermeidungstendenzen bei Lissy, wenngleich ausschließlich bezogen auf die Untersuchungssituation. Sie fürchtete sich z.B., sich "peinlich und albern" zu benehmen oder das Pflegepersonal unflätig zu behandeln, und gab an, sich nicht untersuchen lassen zu wollen. Die Eltern fürchteten ein Wiederauftreten der Angstsymptomatik. Es handelte sich um diejenige Untersuchung, die als auslösendes Ereignis für die massive Angstreaktion von Lissy gewertet wurde. Daher wollten Lissy und ihre Eltern die "Angst vor dem MRT" bewältigen, auch um die bisherigen Behandlungserfolge nicht zu gefährden (Minderung des Rückfallrisikos). Dieser Behandlungsabschnitt fand 
in der Hochschulambulanz statt. Dazu wurde der Untersuchungsablauf zunächst im Spiel (Abb. 2) betrachtet, basierend auf Vorbefunden und Erinnerungen aller Beteiligten. Es wurde ein Szenario der Untersuchung entwickelt und eingeübt.

Anschließend erfolgte einmalig die Beobachtung bei der Vorbereitung einer ähnlichen Untersuchung in einer Arztpraxis mit MRT. Darauf folgte die wiederholte Exposition in vivo an einem Mock-Scanner, basierend auf dem zuvor im Spiel eingeübten Szenario. Der Verlauf der Expositionsübungen folgte dem bekannten regelhaften $\mathrm{Ab}$ lauf. Lissy konnte der Untersuchung zustimmen, sich weitestgehend angstfrei mit Unterstützung ihrer Helferfigur Til Tiger und des Vaters dem Scanner nähern, die Untersuchungsposition für ein Schädel-MRT einnehmen und sich den anterioren Teil der Kopfspule aufsetzen lassen. Dieser Ablauf wurde mehrfach wiederholt und stichpunktartig verschriftlicht. Die eigentliche Untersuchung fand in Abwesenheit der Therapeutin statt; die Kollegen vor Ort gestalteten die Untersuchung anhand des übermittelten verschriftlichten Ablaufs ähnlich dem entwickelten Szenario, sodass die Untersuchung letztlich ohne einen massiven Anstieg der Erregung durchgeführt werden konnte. In der Rückschau bewerteten Lissy und die Eltern die MRT-Untersuchung als sicher bewältigt.

\section{Zweite Ergänzung zum Behandlungsplan: Hilfe zur Selbsthilfe (45.-64. Behandlungseinheit)}

Nach der ersten Behandlungsphase und der erfolgreich bewältigten MRT-Untersuchung setzte eine Erhaltungsphase ein, in der die Bewältigung von Alltagssituationen weiter selbstständig geübt wurde. Im weiteren Verlauf wurde Lissy die Differenz zwischen ihrem körperlichen, sozioemotionalen und intellektuellen Entwicklungsstand (Lebens- und Entwicklungsalter) zunehmend bewusst: So wie sie ihre Erfolge im "Training gegen die Angst” erkannte, erkannte sie auch Situationen im Alltag, in denen sie sich hilflos fühlte. So sorgte sie sich z.B. darum, wie sie mit durchaus möglichen Ereignissen (Erkrankung, Versterben von Familienmitgliedern oder Haustieren, selbst zu erkranken) umgehen könne ("Was ist, wenn ...”). In der Folge zeigte Lissy ein ausgeprägtes Vermeidungs- und Rückversicherungsverhalten (z.B. Besuche bei Großeltern vermeiden; häufiges Nachfragen bei den Eltern, ohne dass deren Erklärungen beruhigen konnten) und es bestand die Gefahr einer ängstlichen oder depressiven Reaktion mit Selbstwertminderung und Verunsicherung. Daher erfolgte die Umwandlung in eine Langzeitbehandlung mit dem Ziel der Vermittlung von Problemlösefertigkeiten. Die Elternarbeit fokussierte die elterliche Anerkennung der Entwicklungs- und Lernfähigkeit des Kindes ("Du kannst das machen" statt "Wir

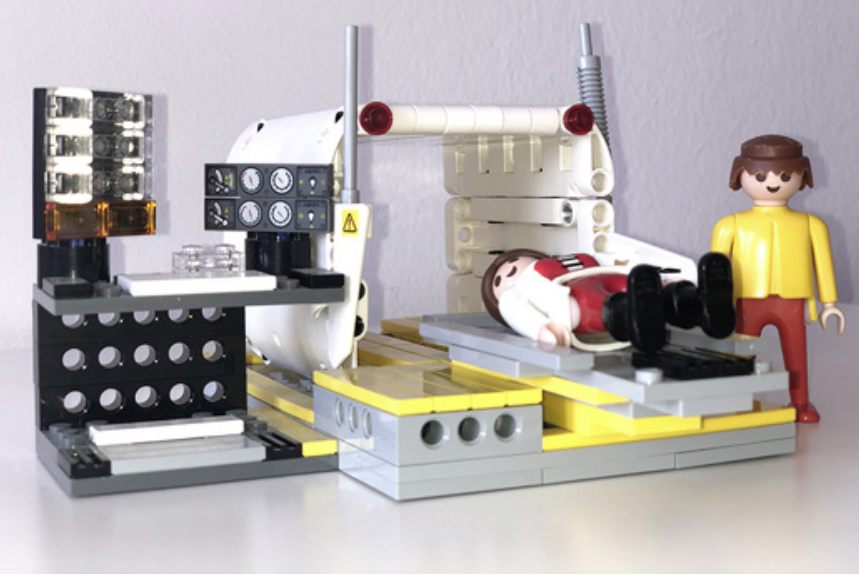

Abb. 2. Szenario zum Untersuchungsablauf im MRT. In der Anordnung liegt Lissy auf dem Untersuchungstisch, der Vater steht aufgrund der Hemianopsie links von ihr.

müssen sehen, wie du zurechtkommen kannst"). Die Behandlungstermine fanden auch hierfür ausnahmslos in der Hochschulambulanz statt und es konnten auch kognitiv orientierte Techniken eingesetzt werden zur Identifikation und Klärung von Annahmen oder Erwartungen der Patientin (“Was könnte denn dann passieren?"), wie direkte Exploration durch Nachfragen und Konkretisieren in der (antizipierten) Problemsituation ("Woher weißt du das?”), Überprüfung der Evidenz (z.B. anhand altersgerechter Medien), Ermunterung zur Reattribution (“es könnte auch ganz anders sein") sowie Erarbeiten rationaler Alternativen und schließlich Einüben neuer Gedanken (häufiger: konkreter Verhaltensweisen) im Spiel, Rollenspiel oder in vivo. Lissy erstellte parallel ein sogenanntes Mutbuch, vergleichbar einer Broschüre, die auf der linken Seite ihre Ängste in Form von Zeichnungen, Bildern oder Texten zeigte und auf der rechten Seite angemessene Gedanken und Verhaltensweisen. Das Mutbuch enthielt Themen zu körperlichen Vorgängen, Erkrankungen oder Versterben wichtiger Bezugspersonen, Verhalten in Notfallsituationen und zur Bewältigung des Schulweges. Kinderbücher, Videos und Spielmaterialien wurden genutzt, um eine vertiefte Auseinandersetzung mit den angstbesetzen Inhalten herbeizuführen und zugleich alternative (hilfreiche) Kognition und Verhaltensweisen zu identifizieren.

\section{Diskussion und Schlussfolgerung}

Der hier vorgestellte Behandlungsfall bedient sich der großen Bandbreite kognitiv-behavioraler Behandlungstechniken in der Kinder- und Jugendlichenpsychotherapie und illustriert die erfolgreiche Anwendung bei einem 
10-jährigen Mädchen mit einer Angststörung bei gleichzeitiger Entwicklungsstörung und Intelligenzminderung. Während der erste und zweite Behandlungsabschnitt einem handlungsbezogenen Vorgehen folgte und weniger kognitiv orientiert war, konnten im dritten Behandlungsabschnitt - auch aufgrund der zunehmenden Reifung des Kindes - verstärkt sprachreiche und kognitiv anspruchsvolle Techniken eingesetzt werden.

Vorgehen und Verlauf der Behandlung waren trotz dieser Besonderheiten ähnlich einer TAU-Behandlung ("treatment as usual"): individuelles, planvolles Vorgehen und Auswahl der Techniken anhand der Beschwerden und Funktionalitäten, Vermeidung paralleler Interventionen, altersangemessene Visualisierung von Therapiezielen, -schritten und -erfolgen. Besonderes Augenmerk galt den spezifischen Wahrnehmungsbedingungen von Lissy. So erfolgte die Ansprache stets von gegenüber oder links von ihr (Hemianopsie). Überraschenderweise gestaltete sich das Antragsverfahren als unkompliziert, trotz der differentialdiagnostischen Einordnung, der BNS-Epilepsie als relative Kontraindikation für ein expositionsorientiertes Vorgehen, der Durchführung im häuslichen Setting und in Beantragung von Doppelsitzungen. Organisatorische (zeitliche) Besonderheiten bestanden im häuslichen Setting während des ersten Therapieabschnittes, hinsichtlich eines stärker systemorientierten Vorgehens zur Unterstützung und Anleitung der Bezugspersonen. Eine Psychoedukation, wie sie aus vergleichbaren Behandlungen bekannt ist, fand hier nicht statt. Möglicherweise waren die Inhalte zu abstrakt für Lissy; ihr Interesse galt vorrangig dem "Mutig werden" und weniger darin "die Angst zu verstehen". Die Auseinandersetzung mit angstbesetzen Situationen war (zumindest zu Behandlungsbeginn) von Minutenangaben begleitet. Dies mag auf den ersten Blick als Sicherheitssignal erscheinen. Allerdings fehlte Lissy zum damaligen Zeitpunkt eine Vorstellung von der Zeit. Zwar hatte sie ein Verständnis für Mengen, Jahreszeiten, Wochentage und Tageszeiten, konnte jedoch nicht die Uhr lesen oder hatte ein Verständnis von Minuten (im Verhältnis zu Stunden etc.). Sie hatte ein Verständnis für den Zahlenraum und konnte zählen, und wußte daher z.B. 5 ist mehr als 3. Die tatsächliche Dauer variierte und es wurde auch keine Stoppuhr o.ä. eingesetzt, die als Sicherheitssignal hätte dienen können. Zum zweiten war nicht sicher, ob nicht durch eine prolongierte Konfrontation mit der angstauslösenden Situation eine massive Angstreaktion oder im ungünstigsten Fall gar ein erneuter epileptischer Anfall ausgelöst würde. Um dieses Risiko zu reduzieren, wurden angstbesetzte Situationen schrittweise und mit Angabe einer Zeitangabe aufgesucht, mit dem Ziel des Aufsuchens und der Bewältigung der Situation, und nicht dem Ziel der maximalen Angstreaktion. Dies entspricht nicht zuletzt auch Befunden zur Expositionstherapie bei Kin- dern und Jugendlichen [Peterman et al., 2019] und Erwachsenen [Craske et al., 2008], nach denen das Ausmaß der initialen oder maximalen Furchtreaktion kein Prädiktor für den Behandlungserfolg ist. Die Termine waren tagesformabhängig unterschiedlich lang, zwischen 30 und $90 \mathrm{~min}$, angesichts schwankender Aufmerksamkeitsund Konzentrationsspannen, und wurden auch angesichts der Wegzeiten als randständige Termine am Nachmittag vereinbart. Die Teilhabe der Therapeutin am häuslichen Alltag der Patientin formte eine belastbare Therapeut-Patient-Beziehung, wenngleich die notwendigen Grenzen einzuhalten waren. Damit verbunden waren aber auch Wegekosten, also nicht abrechenbare Leistungen nach dem einheitlichen Bewertungsmaßstab. Auch der Beratungsbedarf der Bezugspersonen konnte nur mäßig in den Vergütungsrichtlinien abgebildet werden. Zusätzliche Regelungen nach der Änderung der Psychotherapie-Richtlinie vom Oktober 2018 erbrachten diesbezüglich wesentliche Verbesserungen, z.B. zur Abrechenbarkeit zusätzlicher Einheiten (z.B. Sprechstunde, diagnostische Einschätzung, Einbezug von Bezugspersonen [Gemeinsamer Bundesauschuss, 2018]).

Einschränkend muss bedacht werden, dass die geistige Behinderung (hier: Intelligenzminderung) von Lissy mittelgradig ausgeprägt war; eine Übertragung des Vorgehens, der Ergebnisse und prognostischen Aussagen auf Kinder- und Jugendlichenpsychotherapien bei schweren Formen der geistigen Behinderung ist fraglich. Die Indikationsprüfung wurde sowohl durch den behandelnden Neurologen als auch den Gutachter gestützt; dennoch gilt es relative Kontraindikationen (hier: BNS-Epilepsie) sorgfältig abzuwägen; die Konfrontation mit angstauslösenden Situationen gelang hier kleinschrittig und bei Anfallsfreiheit. Streng genommen hätten das verminderte Intelligenzniveau, die BNS-Epilepsie in der Vorgeschichte, die Einschränkungen in den sprachlichen Fertigkeiten und im Reflexionsvermögen ausreichen können, ein anderes, nicht richtlinienorientiertes Vorgehen zu wählen oder die Behandlung abzulehnen.

Spezifische Diagnoseinstrumente für psychische Beschwerden bei Menschen mit geistiger Behinderung existieren kaum [Sarimski, 2007]. Anders als im Routine-Fall wurde hier nur das BAV-3 zur Selbstauskunft eingesetzt, unter der Annahme begrenzter Introspektions- und Verbalisierungsfähigkeiten und der bekannten reduzierten Lesefertigkeiten. Die Mehrheit diagnostischer Informationen entstammten Aussagen der Eltern, Befunden der Mitbehandler und der Verhaltensbeobachtung im häuslichen Setting. Diese hätte durchaus stärker durch diagnostische Instrumente gestützt werden können, z.B. durch den Einsatz symptomübergreifender Inventare zur Fremdanamnese wie die CBCL oder der SDQ. Die Interpretation der Befunde muss dann (wie in jedem anderen Fall auch) im Hinblick auf die Normen (die unabhängig 
vom Intelligenzniveau gelten) vor dem Hintergrund individueller Besonderheiten erfolgen. So wäre es denkbar, die Auswahl der Altersnormen nicht anhand des biologischen Alters sondern anhand des Entwicklungsalters des Kindes zu orientieren.

Die Umsetzung im häuslichen Setting, die variable Termingestaltung sowie die Intensität (Frequenz) der Behandlungskontakte mögen nicht die typische Regelversorgung abbilden; nicht selten sind Behandler nur ungenügend mit den gesundheitlichen Besonderheiten von Menschen mit geistiger Behinderung vertraut oder in der Lage, unter den bestehenden personellen, wirtschaftlichen und fachlichen Rahmenbedingungen den Erfordernissen und Besonderheiten ihrer gesundheitlichen Versorgung Rechnung zu tragen. Zudem ist die Ausgestaltung einer Psychotherapie für Patienten mit geistiger Behinderung in der Aus- und Fortbildung wenig berücksichtigt. Dies muss nicht zwingend ein Mangel sein; der beschriebene Fall dokumentiert die flexible Nutzung von bekannten psychotherapeutischen Methoden und die Adaptation an die Bedürfnisse der Patientin. Demnach ist die psychotherapeutische Behandlung von Kindern und Jugendlichen mit geistiger Behinderung durchaus möglich und - nach Janßen [2018] - vielleicht auch gar nicht schwer. Es ist anzunehmen, dass das Erkennen und die Auseinandersetzung mit dem "Anderssein" und den verfügbaren Alltagskompetenzen gleichermaßen Entwicklungsrisiko und Entwicklungschancen bergen, die durch eine psychotherapeutische Begleitung gut bewältigt werden können. Katamnestisch berichtete die Kindsmutter im Oktober 2020 über stabile Behandlungsergebnisse und eine weitere Ausformung der Alltagskompetenzen: Lissy gelingt die Nutzung öffentlicher Verkehrsmittel und sie kann einzelne Abende auch ohne familiäre Unterstützung allein zu Hause verbringen; beides haben Lis- sy und ihre Eltern analog zum Vorgehen in der Behandlung schrittweise trainiert. Zudem wurde eine weitere MRT-Untersuchung ohne umfangreiche Vorbereitungen erfolgreich bewältigt. Daher erscheint die psychotherapeutische Behandlung auch das langfristige Ziel einer Verhinderung der Manifestation (Eskalation) von weiterem Problemverhalten erreicht zu haben.

\section{Statement of Ethics}

Die Autorin bestätigt die Einhaltung der aktuellen Fassung der Deklaration von Helsinki. Die psychotherapeutische Behandlung wurde lege artis durchgeführt und dokumentiert. Die personenbezogenen Originaldaten (Patientenakte) wird gemäß den derzeit gültigen deutschen datenschutzrechtlichen Bestimmungen aufbewahrt. Identifizierende Personenmerkmale wurden für die Falldarstellung geändert. Die Patientin und ihre Familie haben der Veröffentlichung der Falldarstellung (diesem Artikel) zugestimmt. Die Einholung einer zustimmenden Bewertung der örtlichen Ethikkommission wurde nicht benötigt.

\section{Conflict of Interest Statement}

Die Autorin erklärt, dass kein Interessenkonflikt besteht.

\section{Funding Sources}

Nicht zutreffend. Die Autorin führte die hier beschriebene psychotherapeutische Behandlung im Rahmen der gesetzlichen Regelversorgung durch.

\section{Author Contribution}

S.K. erstellte das Manuskript.

\section{Literatur}

Ahrens-Eipper S, Leplow B. Mutig werden mit Til Tiger. Ein Trainingsprogramm für sozial unsichere Kinder. Göttingen: Hogrefe; 2004.

Ali A, Hall I, Blickwedel J, et al. Behavioural and cognitive-behavioural interventions for outwardly directed aggressive behaviour in people with intellectual disabilities. Cochrane Database Syst Rev. 2015;4:CD003406.

Bergmann F. Verhaltenstherapie bei jungen Menschen mit kognitiven Einschränkungen. Göttingen: Hogrefe; 2019.

Craske MG, Kircanski K, Zelikowsky M, Mystkowski J, Chowdhury N, Baker A. Optimizing inhibitory learning during exposure therapy. Behav Res Ther. 2008 Jan;46(1):5-27.

Deb S, Thomas M, Bright C. Mental disorder in adults with intellectual disability. 1: prevalence of functional psychiatric illness among a community-based population aged between 16 and 64 years. J Intellect Disabil Res. 2001 Dec;45(Pt 6):495-505.
DGKJP - Deutsche Gesellschaft für Kinder- und Jugendpsychiatrie. Psychosomatik und Psychotherapie. S2k Praxisleitlinie Intelligenzminderung. 2014. Verfügbar unter: www.awmf. org/uploads/tx_szleitlinien/028-042l_S2k_Intelligenzminderung_2014-12.pdf, 2014.

Didden R, Korzilius H, van Oorsouw W, Sturmey P. Behavioral treatment of challenging behaviors in individuals with mild mental retardation: meta-analysis of single-subject research. Am J Ment Retard. 2006 Jul;111(4):290-8.

Elstner S. Psychotherapie in der wissenschaftlichen Literatur. In: Glasenapp J, Schäper S, Herausgeber. Barrierefreie Psychotherapie, Möglichkeiten und Grenzen psychotherapeutischer Versorgung von Menschen mit Intelligenzminderung. Dokumentation der Tagung der Deutschen Gesellschaft für seelische Gesundheit geistig Behinderter (DGSGB), Berlin, 2016. Verfügbar unter: https://dgsgb.de/downloads/materialien/Band37.pdf [zitiert 16. November 2020].
Gemeinsamer Bundesausschuss. Beschluss des gemeinsamen Bundesausschusses über eine Änderung der Psychotherapie-Richtlinie: Zusätzliche Regelungen für Menschen mit einer geistigen Behinderung. Pressemitteilung vom 18. Oktober 2018. Verfügbar unter: https://www.g-ba.de/informationen/beschluesse/3528/ [zitiert 25. April 2019].

Hennicke K, Herausgeber. Praxis der Psychotherapie bei erwachsenen Menschen mit geistiger Behinderung. Marburg: Bundesvereinigung Lebenshilfe; 2011.

Hennicke K. Zur psychosozialen Versorgungssituation geistig behinderter Menschen mit zusätzlichen Verhaltensauffälligkeiten und psychischen Störungen. In: Bienstein P, Rojahn J, editors. Selbstverletzendes Verhalten bei Menschen mit geistiger Behinderung. Grundlagen, Diagnostik und Intervention. Göttingen: Hogrefe; 2013. p. 228-50. 
Jäckel A, Simon T. Inhalte zur Psychotherapie bei Kindern und Jugendlichen mit In-telligenzminderung in der Ausbildung zum Kinderund Jugendlichenpsychotherapeuten. Eine Analyse von Ausbildungscurricula. Psychotherapeutenjournal. 2014; 2:131-3.

Janssen CGC, Schuengel C, Stolk J. Understanding challenging behaviour in people with severe and profound intellectual disability: a stress-attachment model. Intellect Disabil Res. 2002;46(6):445-53.

Janßen C. Psychotherapie mit Menschen geistiger Behinderung. Besonderheiten einer vernachlässigten Klientel in der Praxis. Psychotherapeutenjournal. 2018;17(4):337-44.

Jennett HK, Hagopian LP. Identifying empirically supported treatments for phobic avoidance in individuals with intellectual disabilities. Behav Ther. 2008 Jun;39(2):151-61.

Mackowiak K, Lengning A. BAV 3-11. Das Bochumer Angstverfahren für Kinder im Vorschul- und Grundschulalter. Göttingen: Hogrefe; 2010.

Metaxas C, Wünsch A, Simon T, et al. Ambulante Psychotherapie für Kinder und Jugendliche mit Intelligenzminderung. Zur aktuellen Versorgungslage in Baden-Württemberg. Psychotherapeutenjournal. 2014;2:122-30.

Mevissen L, de Jongh A. PTSD and its treatment in people with intellectual disabilities: a review of the literature. Clin Psychol Rev. 2010 Apr;30(3):308-16.
Peterman JS, Carper MM, Kendall PC. Testing the habituation-based model of exposures for child and adolescent anxiety. J Clin Child Adolesc Psychol. 2019;48 Suppl 1:S34-44.

Prout HAT, Browning BK. The effectiveness of psychotherapy with persons with intellectual disabilities. In: Fletcher RJ, editor. Psychotherapy for individuals with intellectual disability. Kingston: NADD Press; 2011. p. 26587.

Remschmidt HM, Schmidt F, Poustka F. Multiaxiales Klassifikationsschema für psychische Störungen des Kindes- und Jugendalters nach ICD-10 der WHO. 4th ed. Bern: Huber; 2016.

Robertson B. The adaptation and application of mindfulness-based psychotherapeutic practices for individuals with intellectual disabilities. Adv Ment Health Intellect Disabil. 2011;5(5):46-52.

Sappok T, Voß T, Millauer E, Schade C, Diefenbacher A. Psychotherapie bei Menschen mit Intelligenzminderung. Theoretischer Hintergrund und praktische Umsetzung. Nervenarzt. 2010;81:827-36.

Sarimski K. Psychische Störungen bei behinderten Kindern und Jugendlichen - Übersicht und Schlussfolgerungen für die Psychodiagnostik. Z Kinder Jugendpsychiatr Psychother. 2007 Jan;35(1):19-29.

Schützwohl M, Koch A, Koslowski N, Puschner B, Voß E, Salize HJ, et al. Mental illness, problem behaviour, needs and service use in adults with intellectual disability. Soc Psychiatry Psychiatr Epidemiol. 2016 May;51(5):767-76.
Sparrow SS, Balla DA, Cicchetti DV. Vineland Adaptive Behavior Scale. A revision of the Vineland Social Maturity Scale by Edbar A. Doll. Survey Form Manual. Circle Pines: American Guidance Service; 1984.

Stünkel-Grees N, Clausen J, Wünsch A. Ambulante Psychotherapie für Kinder und Jugendliche mit geistiger Behinderung. Prax Kinderpsychol Kinderpsychiatr. 2018 Mar;67(3):224-38.

Sturmey P. Treatment of psychopathology in people with intellectual and other disabilities. Can J Psychiatry. 2012 Oct;57(10):593-600.

Tewes U, Schallberger P, Rossmann U, editors. Hamburg-Wechsler-Intelligenztest für Kinder III (HAWIK-III). Bern: Huber; 1999.

Vereenooghe L, Langdon PE. Psychological therapies for people with intellectual disabilities: a systematic review and meta-analysis. Res Dev Disabil. 2013 Nov;34(11):4085-102.

Werther F. Warum finden Menschen mit geistiger Behinderung so schwer einen ambulanten Psychotherapieplatz? Überlegungen $\mathrm{zu}$ den Ursachen und Gedanken zur Überwindung der Misere. Psychotherapeutenjournal. 2005;4(2):116-22.

Willner P. The effectiveness of psychotherapeutic interventions for people with learning disabilities: a critical overview. Intellect Disabil Res. 2005 Jan;49(Pt 1):73-85.

Zeiger T, Petrovic-Dovat L, Bansal PS. Exposure therapy with a non-verbal child: a case report. Athens J Soc Sci. 2017;4(2):137-46. 